Zhen J. Wang, MD

Fergus V. Coakley, MD

Yanjun Fu, PhD

Bonnie N. Joe, MD, PhD

Sven Prevrhal, PhD

Luis A. Landeras, MD

Emma M. Webb, MD

Benjamin M. Yeh, MD

\title{
Renal Cyst Pseudoenhancement
} at Multidetector CT: What Are the

\section{Effects of Number of Detectors and}

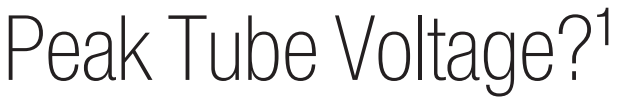

Materials and Methods:

Results:

Conclusion:
${ }^{1}$ From the Department of Radiology, University of California-San Francisco, 505 Parnassus Ave, Box 0628, C-372C, San Francisco, CA 94143-0628. From the 2007 RSNA Annual Meeting. Received September 7, 2007; revision requested December 12; revision received December 16; final version accepted January 28, 2008. Z.J.W. supported in part by National Institute of Biomedical Imaging and Bioengineering Training Grant 1 T32 EB001631-01A1. Address correspondence to Z.J.W. (e-mail: jane.wang@radiology.ucsf.edu).
Purpose:

To determine the effect of the number of detectors and peak tube voltage on renal cyst pseudoenhancement in a phantom model.

This study on computed tomographic (CT) phantoms did not require institutional review board approval. The renal compartments of a CT phantom were filled with iodinated contrast material diluted to attain attenuations of 40, 140, and $240 \mathrm{HU}$. Saline-filled cylinders simulating cysts of varying diameters (range, $0.7-3.0 \mathrm{~cm}$ ) were serially suspended in the renal compartments and scanned at 80, 90, 100, 120 , and $140 \mathrm{kVp}$ in 16-detector $(n=3)$ and 64-detector $(n=2)$ CT scanners. Generalized estimating equations were used to determine predictors of cyst pseudoenhancement (defined as a $>10 \mathrm{HU}$ increase in cyst attenuation when the background renal attenuation increased from 40 to 140 or $240 \mathrm{HU})$.

Pseudoenhancement was seen with higher frequency (59 [61\%] of 96 cysts vs 52 [39\%] of 132 cysts, $P<.05)$ and magnitude (17 vs $13 \mathrm{HU}, P<.005)$ with 64 - rather than with 16-detector scanners. Pseudoenhancement was also seen with higher frequency (25 [42\%] of 60 cysts vs 11 [18\%] of 60 cysts, $P<.005$ ) and magnitude (18 vs $13 \mathrm{HU}$, $P<.05)$ at $140 \mathrm{kVp}$ than at 80 or $90 \mathrm{kVp}$. Cyst pseudoenhancement increased with higher background renal enhancement $(P<.005)$ and smaller cyst diameter $(P<$ $.05)$. The number of detectors, peak tube voltage, renal parenchymal enhancement level, and cyst diameter were independent predictors of cyst pseudoenhancement.

Lower tube voltage settings may be useful when accurate differentiation between small renal cysts and solid masses is critical, particularly for 64-detector CT scanners.

๑ RSNA, 2008 
$\mathbf{T}$ he distinction between solid and cystic masses seen at computed tomography (CT), which is a particularly common and important issue in evaluating focal renal lesions, is determined primarily on the evaluation of whether the lesion enhances after the intravenous administration of iodinated contrast material. While this approach is generally robust, the assessment of enhancement in small renal cystic lesions may be unreliable. Attenuation measurements for such small lesions may erroneously suggest enhancement even though the lesion is truly cystic because of partial volume averaging and pseudoenhancement. Renal cyst pseudoenhancement refers to the artifactual increase in the attenuation of a simple renal cyst following contrast material administration, even when the effects of partial volume averaging have been removed (1-4). Pseudoenhancement is most problematic for small cysts and at high levels of renal parenchymal enhancement $(1-3,5-7)$. This problem is magnified by the fact that renal cysts are present in $20 \%-40 \%$ of the population (8) and that certain renal cell carcinomas, such as the papillary subtype, typically enhance in a weak and homogeneous manner (9). Pseudoenhancement may therefore lead to mischaracterization of a small renal cyst as an enhancing neoplasm and unnecessary intervention.

Pseudoenhancement is believed to be a consequence of beam-hardening effects of the enhanced renal parenchyma combined with artifact introduced by the CT image reconstruction algorithm (1). A recent report suggests that pseudoenhancement is worse with newer-generation multidetector CT scanners than with earlier scanners (10). Given the increasing prevalence of 16 - and 64detector scanners in clinical practice, confirmation of these findings, as well as improved understanding of the means to reduce pseudoenhancement, are

\section{Advance in Knowledge}

- Renal cyst pseudoenhancement can be reduced by using lower peak tube voltage settings. clearly needed. While it is known that peak tube voltage settings affect CT image contrast and attenuation measurement (11), the influence of peak tube voltage on pseudoenhancement is not well studied. Therefore, we undertook this study to determine the effect of the number of detectors and peak tube voltage on renal cyst pseudoenhancement in a phantom model.

\section{Materials and Methods}

\section{CT Phantom}

A water-filled abdominal CT phantom, measuring approximately $32 \times 27 \mathrm{~cm}$ in cross section and $20 \mathrm{~cm}$ in length, was cast from the abdomen of a male volunteer of average size (12). Two compartments, each measuring $6 \mathrm{~cm}$ in diameter, were placed bilaterally to simulate the kidneys (Fig 1). The walls of the phantom and the renal compartments were made of polyethylene, which has an attenuation approximating that of fat. The renal compartments were sequentially filled with iohexol (Omnipaque 350; Nycomed-Amersham, Princeton, NJ) of three different dilutions, selected to reflect the established range of renal attenuation (unenhanced, $40 \mathrm{HU}$; moderately enhanced, $140 \mathrm{HU}$; and maximally enhanced, $240 \mathrm{HU})$. Since iodine is known to have higher attenuation at low peak tube voltage settings (11), the dilution was adjusted for each peak tube voltage used in the study $(80,90,100$, 120 , and $140 \mathrm{kVp}$ ) to maintain the same renal background attenuation $(40,140$, and $240 \mathrm{HU}$ ) for the different tube voltages (Table 1). To simulate renal cysts, saline-filled polyethylene cylinders of six diameters $(0.7,1.0,1.3,1.5,2.1$, and $3.0 \mathrm{~cm}$ ) were constructed. The cylinders measured $8 \mathrm{~cm}$ in length. The cylindrical shape of the cysts eliminated

\section{Implication for Patient Care}

- Lower tube voltage settings may prove useful when accurate differentiation between small renal cysts and solid masses is critical, particularly for 64-detector CT scanners. the effect of partial volume averaging for our study. The saline-filled simulated renal cysts had an attenuation of approximately $5 \mathrm{HU}$ when immersed in saline baths.

\section{Imaging Techniques}

The six simulated renal cysts were sequentially suspended in the renal compartments of the phantom (Fig 2), and scanned in five multidetector CT scanners (16 detector, $n=3 ; 64$ detector, $n=2$ ) at different peak tube voltage settings $(80,90,100,120$, and $140 \mathrm{kVp})$ and constantly at $200 \mathrm{~mA}$ tube current. The scanning parameters are shown in Table 2. Three 16-detector scanners (Lightspeed, GE Healthcare, Madison, Wis $[n=1]$; Somatom Sensation, Siemens Medical Solutions, Forchheim, Germany $[n=1]$; and Mx8000, Philips Medical Systems, Best, the Netherlands $[n=1])$ and two 64-detector scanners (Lightspeed VCT, GE Healthcare) were used. For the GE and Siemens scanners, the cysts were scanned at 80, 100, 120, and $140 \mathrm{kVp}$. For the Philips scanner, the cysts were scanned at 90,120, and $140 \mathrm{kVp}$ (80- and $100-\mathrm{kVp}$ settings were not available on this scanner). For quality control, each scanner was calibrated immediately prior to the experiments according to manufacturer's specifications. The renal cyst phantoms were scanned in saline baths immediately before and after each experiment, documenting absence of iodine contamination. The criterion used for absence of

\section{Published online before prin} 10.1148/radiol.2482071583

\section{Radiology 2008; 248:910-916}

Abbreviation:

$\mathrm{ROI}=$ region of interest

\section{Author contributions:}

Guarantors of integrity of entire study, Z.J.W., F.V.C., B.M.Y.; study concepts/study design or data acquisition or data analysis/interpretation, all authors; manuscript drafting or manuscript revision for important intellectual content, all authors; approval of final version of submitted manuscript, all authors; literature research, Z.J.W., F.V.C., B.N.J., S.P., L.A.L., E.M.W., B.M.Y.; experimental studies, Z.J.W., F.V.C., Y.F., B.M.Y.; statistical analysis, Z.J.W., B.M.Y.; and manuscript editing, all authors

Authors stated no financial relationship to disclose. 
iodine contamination was a change in cyst attenuation of $5 \mathrm{HU}$ or less; no iodine contamination occurred in any of the renal cyst phantoms in our study.

\section{Image Analysis}

The primary investigator (Z.J.W.) reviewed all CT images on a picture archiving and communication system workstation (Impax; Agfa, Mortsel, Belgium). The images from the same CT scanner were reviewed generally in the order in which they were acquired (ie, with increasing renal background attenuation and increasing tube voltage settings). The images were reviewed over a period of 2 months. The attenuation of each simulated cyst was the mean of three manually placed circular regions of interest (ROIs) on contiguous images in the mid portion of the cylindrical cyst, with care to exclude the cyst walls. The ROIs were approximately 0.17 , $0.35,0.59,0.79,1.53$, and $3.14 \mathrm{~cm}^{2}$ for the 0.7-, 1.0-, 1.3-, 1.5-, 2.1-, and $3.0-\mathrm{cm}$ diameter cylindrical cysts, respectively. The ROIs were placed at approximately the same location for each cyst. A total of 1026 ROI measurements (three for each mean cyst attenuation measurement) were acquired for this study, resulting in 342 mean cyst attenuation measurements, which sampled a combination of peak tube voltages, scanner types, cyst diameters, and background renal attenuation levels. There were six simulated cysts of different diameters, scanned at four peak tube voltages $(80,100,120$, and 140 $\mathrm{kVp}$ ) in four scanners (the GE and Siemens scanners), at three peak tube voltages $(90,120$, and $140 \mathrm{kvp})$ in one scanner (the Philips scanner), and at three renal background attenuation levels, resulting in 342 mean cyst attenuation measurements ( 6 cysts $\times 4$ peak tube voltages $\times 3$ renal background attenuation levels $\times 4$ scanners $]+[6$ cysts $\times 3$ peak tube voltages $\times 3$ renal background attenuation levels $\times 1$ scanner] $=342$ ) (Fig 3). Cyst enhancement was determined by measuring the difference in cyst attenuation values between the 140 - and $40-\mathrm{HU}$ renal background attenuation levels and between the 240and 40-HU renal background attenua- tion levels. Therefore, a total of 228 measurements of cyst enhancement were obtained ( 6 cysts $\times 4$ peak tube voltages $\times 2$ changes in renal background enhancement $\times 4$ scanners] + $[6$ cysts $\times 3$ peak tube voltages $\times 2$ changes in renal background enhancement $\times 1$ scanner] = 228) (Fig 4). Pseudoenhancement was considered to be present when the cyst attenuation level increased by more than $10 \mathrm{HU}$ $(13,14)$.

\section{Statistical Analysis}

Statistical analysis was performed with software (Stata, version 8.0; Stata, College Station, Tex). The $\chi^{2}$ test was used to compare the frequency of cyst pseudoenhancement, and the two-tailed Student $t$ test was used to compare the magnitude of cyst pseudoenhancement between 16- and 64-detector scanners, tube voltage settings (high [140 kVp] vs low [80 and $90 \mathrm{kVp}$ ]), two renal background enhancement levels (140 and $240 \mathrm{HU}$ ), and cyst size (small $[\leq 1.0 \mathrm{~cm}]$ vs large $[>1.0 \mathrm{~cm}])$. To assess for independent predictors of renal cyst pseudoenhancement, generalized estimating equations determined by using a binary logistic regression model (to assess likelihood of finding pseudoenhancement) or a linear regression model (to assess magnitude of pseudoenhancement) were used. Each model included scanner type (16 vs 64 detector), tube voltage settings $(80,90,100,120$, and $140 \mathrm{kVp})$, renal background enhancement levels (140 and $240 \mathrm{HU}$ ), and cyst diameters $(0.7,1.0,1.3,1.5,2.1$, and $3.0 \mathrm{~cm})$. The covariance structure was modeled by assuming data to be correlated or independent when derived from the same or from different imaging sessions, respectively. Results were considered significant for $P$ values of less than .05.

\section{Results}

The frequency and magnitude of cyst pseudoenhancement were significantly greater with 64 - than with 16-detector scanners across all peak tube voltages, background renal enhancement levels, and cyst diameters (frequency, $P<.05$; magnitude, $P<.005$ ) (Fig 5). Fifty-nine $(61 \%)$ of 96 cyst measurements demonstrated a mean pseudoenhancement of $17 \mathrm{HU} \pm 5$ (standard deviation) on the 64-detector scanners, compared with 52 (39\%) of 132 cyst measurements with a mean pseudoenhancement of 13

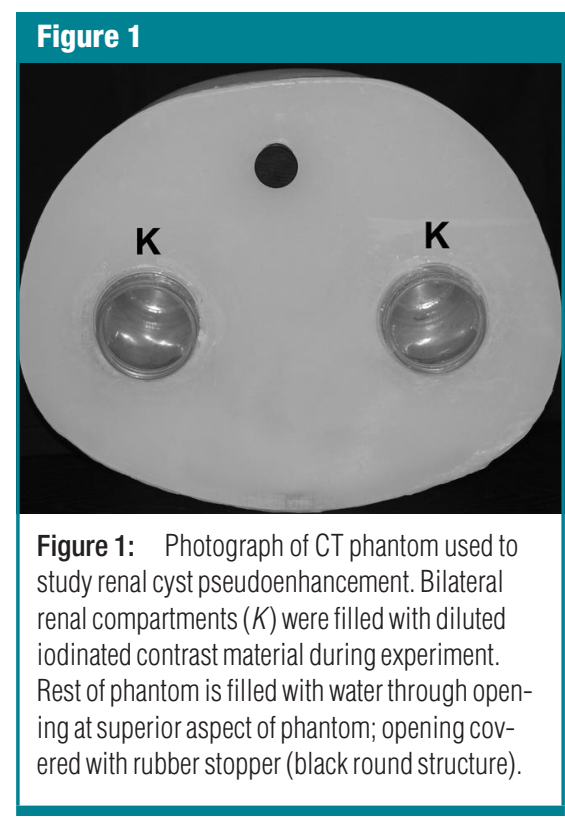


$\mathrm{HU} \pm 3$ on the 16-detector scanners. When pseudoenhancement was compared between different generation multidetector CT scanners from the same manufacturer (GE Healthcare), the frequency of pseudoenhancement increased from 14 (29\%) of 48 cyst measurements on the GE Healthcare 16-detector scanner to $54(56 \%, P<.005)$ of 96 measurements on the GE 64-detector scanners; the magnitude of mean pseudoenhancement increased from $13 \mathrm{HU} \pm 2$ on the GE Healthcare 16-detector scanner to 17 $\mathrm{HU} \pm 5(P<.05)$ on the GE Healthcare 64-detector scanners.

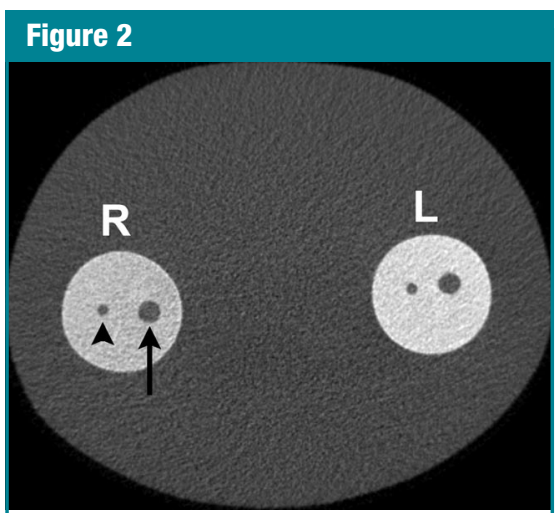

Figure 2: CT scan of phantom shows simulated renal cysts within bilateral renal compartments. Renal compartments were serially filled with dilutions of iodinated contrast material to simulate established range of renal parenchymal attenuation. Saline-filled cylinders were placed in renal compartments $(R=$ right, $L=$ left) to simulate 1.0-cm (arrow) and 0.7-cm (arrowhead) intrarenal cysts.
Higher tube voltage settings resulted in higher frequency and greater magnitude of cyst pseudoenhancement across all scanners, background renal enhancement levels, and cyst diameters (Fig 6). At a tube voltage of $80 \mathrm{kVp}(90$ $\mathrm{kVp}$ for the Philips scanner), pseudoenhancement occurred in only 11 (18\%) of 60 of cyst measurements with a mean magnitude of $13 \mathrm{HU} \pm 4$; however, at $140 \mathrm{kVp}$, pseudoenhancement was seen in a higher proportion of cyst measurements (25 of $60,42 \%, P<.005)$ with a higher mean magnitude of $18 \mathrm{HU} \pm 5$ $(P<.05)$.

Higher renal background attenuation levels and smaller cyst diameters were each significantly associated with increased cyst pseudoenhancement. At $140 \mathrm{HU}$ renal background attenuation, $16(14 \%)$ of 114 cyst measurements showed a mean magnitude of $12 \mathrm{HU} \pm$ 2. In comparison, at $240 \mathrm{HU}$ renal background attenuation, pseudoenhancement was seen in a higher proportion of cyst measurements (62 of 114, 54\%, $P<.001)$ with a higher mean magnitude of $17 \mathrm{HU} \pm 5(P<.005)$. Larger cysts $(>1.0 \mathrm{~cm}$ in diameter) showed pseudoenhancement in 40 (26\%) of 152 cyst measurements, with a mean magnitude of $12 \mathrm{HU} \pm 6$. In comparison, small cysts $(\leq 1.0 \mathrm{~cm})$ showed a significantly higher frequency of pseudoenhancement (30 of 76 measurements, 39\%, $P<.05)$, and a higher mean magnitude of $20 \mathrm{HU} \pm 3(P<.001)$.

Generalized estimating equation models showed that the use of 64- rather than 16-detector scanners, higher peak tube voltage settings, higher renal background attenuation levels, and smaller cyst diameters were each independent predictors of both the likelihood of finding pseudoenhancement $(P<.001, P<$ $.001, P<.001$, and $P<.05$ respectively), and the magnitude of the pseudoenhancement $(P<.001$ for each).

\section{Discussion}

We found that the frequency and magnitude of renal cyst pseudoenhancement were significantly higher for 64 detector scanners, as compared with 16-detector scanners. Our findings are in agreement with a recent study showing increasing incidence of renal cyst pseudoenhancement with newer-generation CT scanners (10) and support the concern for a worsening trend in the reliability of attenuation measurements as the numbers of detectors per scanner increase. While $10 \mathrm{HU}$ was the traditional threshold level for determining renal lesion enhancement (15), higher threshold levels are likely required for multidetector scanners $(1,2,5,10)$, even for different CT reconstruction algorithms from the same multidetector scanner (10). Regarding CT scanners with higher numbers of detectors, equivocal cases of enhancement will likely occur more commonly and referral to magnetic resonance or ultrasonographic imaging for confirmation should be strongly considered. The increased pseudoenhancement with higher numbers of detectors may result from higher

Table 2

\section{Parameters for Multidetector CT Scanners}

\begin{tabular}{|c|c|c|c|c|}
\hline \multirow[b]{2}{*}{ Scan Parameter } & \multicolumn{4}{|c|}{ No. and Type of CT Scanner } \\
\hline & GE Lightspeed $(n=1)$ & GE Lightspeed VCT $(n=2)$ & Philips Mx8000 $(n=1)$ & Siemens Somatom Sensation $(n=1)$ \\
\hline No. of detectors & 16 & 64 & 16 & 16 \\
\hline Peak voltage settings (kVp) & $80,100,120,140$ & $80,100,120,140$ & $90,120,140$ & $80,100,120,140$ \\
\hline Tube current (mA) & 200 & 200 & 200 & 200 \\
\hline Section thickness (mm) & 2.5 & 2.5 & 3 & 3 \\
\hline Reconstruction kernel & Standard & Standard & C & B31S \\
\hline Beam pitch & $1.375: 1$ & $1.375: 1$ & $1.238: 1$ & $1.375: 1$ \\
\hline Table speed (mm per rotation) & 27.5 & 55 & 29.7 & 32.4 \\
\hline Gantry rotation time (sec) & 0.8 & 0.5 & 0.75 & 0.75 \\
\hline Field of view $\left(\mathrm{cm}^{2}\right)$ & 400 & 400 & 400 & 400 \\
\hline
\end{tabular}


intersection scatter radiation and crosstalk owing to the higher number of simultaneously acquired sections (16). CT image reconstruction algorithms may only partially compensate for the scatter radiation, resulting in a varying degree of pseudoenhancement that depends on the reconstruction algorithms.

More importantly, we found that lower peak tube voltage settings were associated with significantly lower frequency and magnitude of cyst pseudoenhancement in our phantom model. This observation may result from two factors. First, the contrast material solutions we used for lower tube voltage settings contained lower concentrations of iodine because less iodine is needed to achieve the same renal background attenuation level at low tube voltage compared with high tube voltage (11). A reduced amount of required iodine results in less beam hardening of the $\mathrm{x}$-ray by the iodine. Although the concomitant lower x-ray beam energy at lower tube voltage may increase beam hardening, the reduction in the amount of required iodine may result in an overall decrease in x-ray beam hardening at lower peak tube voltage and therefore less cyst pseudoenhancement. Second, lower tube voltage settings produce $\mathrm{x}$-rays with energies closer to the $\mathrm{k}$ edge of iodine $(33 \mathrm{keV})$, hence, a greater proportion of $\mathrm{x}$-rays is completely absorbed (photoelectric effect) rather than scattered (Compton interactions) by the iodinated contrast material. The decreased scattered radiation at lower tube voltages may result in fewer nonlinearity errors at CT (16) and therefore less cyst pseudoenhancement. Our results differ slightly from those of Coulam et al (2) who reported increased cyst pseudoenhancement at lower tube voltages. However, this discrepancy is readily explained by that study's use of fixed iodine concentrations across the range of tube voltage settings rather than compensatory reductions in iodine concentrations at lower tube voltages, resulting in an unnecessarily high enhancement of renal parenchyma and higher cyst pseudoenhancement at lower peak tube voltage.
Figure 3

$\begin{array}{cc}\text { GE Lightspeed } & \text { Siemens Sensation } \\ \text { 16-detector } & \text { 16-detector } \\ (n=1) & (n=1) \\ \times & \times \\ \text { Cysts }(n=6) & \text { Cysts }(n=6) \\ \times & \times\end{array}$

Philips Mx8000
16-detector
$(n=1)$
$\times$
Cysts $(n=6)$
$\times$

GE Lightspeed VCT 64-detector $(n=2)$ $\times$

Cysts $(n=6)$ $x$

Tube voltage settings Tube voltage settings Tube voltage settings Tube voltage settings $(n=4)$ $\times$ $(n=4)$ $\times$

$(n=3)$

$\times$

$(n=4)$

Renal background Renal background Renal background Renal background attenuation $(40,140$, attenuation $(40,140$, attenuation $(40,140$, attenuation $(40,140$, and $240 \mathrm{HU}[n=3])$ and $240 \mathrm{HU}[n=3])$ and $240 \mathrm{HU}[n=3])$ and $240 \mathrm{HU}[n=3])$

$\begin{array}{cccc}\downarrow & \downarrow & \downarrow & \downarrow \\ \text { Cyst attenuation } & \text { Cyst attenuation } & \text { Cyst attenuation } & \text { Cyst attenuation } \\ \text { measurement } & \text { measurement } & \text { measurement } & \text { measurement } \\ (n=72) & (n=72) & (n=54) & (n=144)\end{array}$
$(n=72)$ $(n=72)$ $(n=54)$ $(n=144)$

Total cyst attenuation measurements $(n=342)$

Figure 3: Flowchart of study design to obtain total number of cyst attenuation measurements.
At lower peak voltage settings, reduced doses of iodinated contrast material can be used owing to the increased sensitivity of CT for iodine detection, with the added benefit that reductions in contrast levels can reduce the risk of contrast-induced nephropathy (17). In our study, the amount of contrast material required to achieve $140 \mathrm{HU}$ renal background attenuation at $80 \mathrm{kVp}$ was about $50 \%$ of what was required at 140 $\mathrm{kVp}$ (Table 1). An even more compelling benefit of reduced tube voltages for clinical CT scanning is the substantial reduction in radiation dose, which has been shown to be reduced as much as $56 \%$ in abdominal CT (11). These two benefits of lower tube voltage settings, combined with the reduction in pseudoenhancement artifact, suggest that reduced tube voltage settings may prove useful when imaging patients for renal mass characterization at CT. Scanning at lower tube voltage settings, however, results in higher image noise levels, which degrade image quality. 
Therefore, lowering tube voltage may not be possible in the setting of large noise at lower tube voltage settings may be partially compensated for by increasing tube current. Additional studies are needed to determine the optimal tube current when a lower tube voltage setting is used to optimize image quality and to decrease radiation dose.

Our study had several limitations. Ours was an in vitro study and the obpatient body weight. The higher image

served frequency and magnitude of cyst pseudoenhancement may be different among patients. Nevertheless, our phantom study allowed for cyst pseudoenhancement assessment in the absence of motion and partial volume averaging, and in a setting of repeated imaging at different tube voltages, which is not feasible in patients. Second, both 64-detector scanners used in our experiment were manufactured by GE Healthcare; we did not have access

\section{Figure 5}

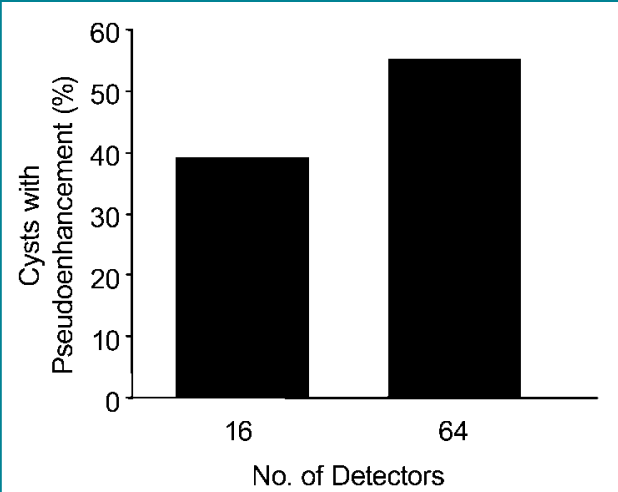

a.

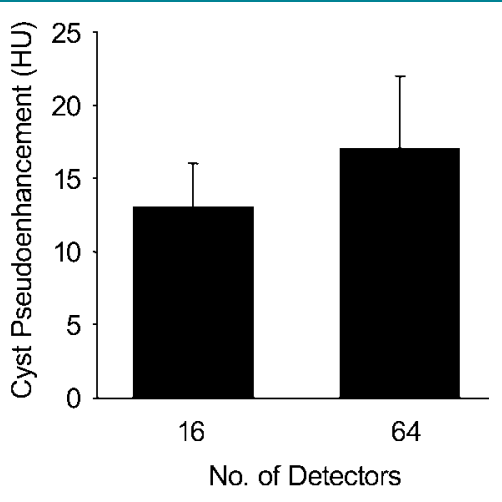

b.

Figure 5: Bar graphs of (a) frequency and (b) magnitude of pseudoenhancement for 16- and 64-detector CT scanners, averaged across all tube voltages, cyst diameters, and background renal enhancement. Frequency and magnitude of cyst pseudoenhancement were significantly higher for 64-detector scanner compared with 16-detector scanners (frequency, $P<.05$; magnitude, $P<.005$ ). T-whiskers demarcate 1 standard deviation from the mean.

\section{Figure 6}
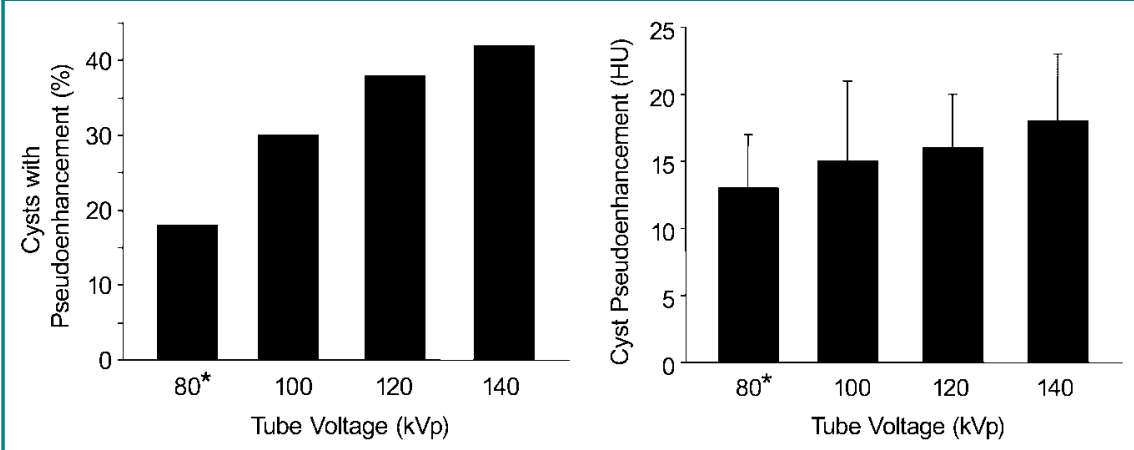

a.

b.

Figure 6: Bar graphs of (a) frequency and (b) magnitude of cyst pseudoenhancement at different peak tube voltages, averaged across all scanners, cyst sizes, and renal background attenuation levels. Lower peak tube voltages of 80 or $90 \mathrm{kVp}$ were associated with significantly lower frequency and magnitude of cyst pseudoenhancement (frequency, $P<.005$; magnitude, $P<.05$ ). $*=$ includes pseudoenhancement measurements at $90 \mathrm{kVp}$ on Philips Mx8000 scanner. T-whiskers demarcate 1 standard deviation from mean. to 64-detector scanners from other manufacturers to make direct comparisons of the degree of cyst pseudoenhancement between the 16 - and 64 detector scanners from other manufacturers. However, our results agree with those from the previous report comparing renal cyst pseudoenhancement between the different generations of multidetector Siemens scanners (10). Third, we did not investigate the effect of reconstruction algorithms on pseudoenhancement in our phantom study. As noted by Birnbaum et al (10), cyst pseudoenhancement was significantly affected by using different reconstruction algorithms, even within the same manufacturer. However, reconstruction algorithms alone did not account for the differences in cyst pseudoenhancement between the 64- and 16-detector scanners in our study, as we also showed significantly higher frequency and magnitude of cyst pseudoenhancement with the 64- and 16-detector GE Healthcare scanners compared by using the same reconstruction algorithms. Furthermore, the reconstruction algorithms selected for our phantom experiments were the ones optimized for our clinical abdominal imaging protocols. Fourth, the investigator who reviewed the CT images could not be completely blinded to the knowledge of the tube voltage settings because the images obtained at lower peak tube voltage tended to have greater visible image noise. Although the knowledge of the tube voltage settings may introduce bias, the use of objective attenuation measurement should minimize that potential bias.

In conclusion, renal cyst pseudoenhancement is more pronounced on 64than on 16-detector CT scanners but can be decreased by using a lower tube voltage setting. Lower tube voltage settings may prove useful when accurate differentiation between small renal cysts and solid masses is critical.

Renal cyst pseudoenhancement worsens with 64-detector rather than with 16-detector CT scanners, higher peak tube voltage setting, higher renal parenchymal enhancement, and smaller cyst size. While radiologists are not readily able to change their CT scanners, low- 
ering the tube voltage setting with a compensatory decrease in intravenous contrast agent dose may prove useful to reduce the pseudoenhancement effect, particularly for the 64-detector CT scanners.

\section{References}

1. Maki DD, Birnbaum BA, Chakraborty DP, Jacobs JE, Carvalho BM, Herman GT. Renal cyst pseudoenhancement: beam-hardening effects on CT numbers. Radiology 1999;213: $468-472$

2. Coulam CH, Sheafor DH, Leder RA, Paulson EK, DeLong DM, Nelson RC. Evaluation of pseudoenhancement of renal cysts during contrast-enhanced CT. AJR Am J Roentgenol 2000;174:493-498.

3. Bae KT, Heiken JP, Siegel CL, Bennett HF. Renal cysts: is attenuation artifactually increased on contrast-enhanced CT images? Radiology 2000;216:792-796.

4. Birnbaum BA, Jacobs JE, Ramchandani P. Multiphasic renal CT: comparison of renal mass enhancement during the corticomedullary and nephrographic phases. Radiology 1996;200:753-758

5. Heneghan JP, Spielmann AL, Sheafor DH,
Kliewer MA, DeLong DM, Nelson RC. Pseudoenhancement of simple renal cysts: a comparison of single and multidetector helical CT. J Comput Assist Tomogr 2002;26: $90-94$.

6. Abdulla C, Kalra MK, Saini S, et al. Pseudoenhancement of simulated renal cysts in a phantom using different multidetector CT scanners. AJR Am J Roentgenol 2002; 179:1473-1476.

7. Birnbaum BA, Maki DD, Chakraborty DP, Jacobs JE, Babb JS. Renal cyst pseudoenhancement: evaluation with an anthropomorphic body CT phantom. Radiology 2002;225:83-90.

8. Tada S, Yamagishi J, Kobayashi H, Hata Y, Kobari T. The incidence of simple renal cyst by computed tomography. Clin Radiol 1983; 34:437-439.

9. Tsuda K, Kinouchi T, Tanikawa G, et al. Imaging characteristics of papillary renal cell carcinoma by computed tomography scan and magnetic resonance imaging. Int J Urol 2005; 12:795-800.

10. Birnbaum BA, Hindman N, Lee J, Babb JS. Renal cyst pseudoenhancement: influence of multidetector CT reconstruction algorithm and scanner type in phantom model. Radiology $2007 ; 244: 767-775$.
11. Nakayama Y, Awai K, Funama Y, et al. Ab dominal CT with low tube voltage preliminary observations about radiation dose, contrast enhancement, image quality, and noise. Radiology 2005;237:945-951.

12. Huda W, Scalzetti EM, Levin G. Technique factors and image quality as functions of pa tient weight at abdominal CT. Radiology 217;2000:430 - 435 .

13. Bosniak MA. The current radiological approach to renal cysts. Radiology 1986;158: $1-10$.

14. Bosniak MA, The small $(\leq 3.0 \mathrm{~cm})$ renal parenchymal tumor: detection, diagnosis, and controversies. Radiology 1991;179:307-317.

15. Bosniak MA, Rofsky NM. Problems in the detection and characterization of small renal masses. Radiology 1996;200:286-287.

16. Vetter JR, Holden JE. Correction for scattered radiation and other background signals in dual-energy computed tomography material thickness measurements. Med Phys 1988;15:726-731.

17. Morcos SK, Thomsen HS, Webb JA. Contrast media-induced nephrotoxicity: a consensus report. Contrast Media Safety Committee, European Society of Urogenital Radiology (ESUR) Eur Radiol 1999;9:1602-1613. 


\section{Radiology 2008}

\section{This is your reprint order form or pro forma invoice}

(Please keep a copy of this document for your records.)

Reprint order forms and purchase orders or prepayments must be received 72 hours after receipt of form either by mail or by fax at 410-820-9765. It is the policy of Cadmus Reprints to issue one invoice per order.

Please print clearly.

Author Name

Title of Article

Issue of Journal

Number of Pages

Color in Article?

Reprint \#

$\mathrm{KB} \#$

Publication Date

Symbol Radiology

Please include the journal name and reprint number or manuscript number on your purchase order or other correspondence.

Order and Shipping Information

Reprint Costs (Please see page 2 of 2 for reprint costs/fees.)

Number of reprints ordered $\$$

Number of color reprints ordered \$

Number of covers ordered

Subtotal $\$$

Taxes

$\$$

(Add appropriate sales tax for Virginia, Maryland, Pennsylvania, and the District of Columbia or Canadian GST to the reprints if your order is to be shipped to these locations.)

First address included, add \$32 for each additional shipping address

TOTAL \$
Shipping Address (cannot ship to a P.O. Box) Please Print Clearly

Name

Institution

Street

City _ State __ Zip

Country

Quantity Fax

Phone: Day

E-mail Address Evening

Additional Shipping Address* (cannot ship to a P.O. Box)

Name

Institution

Street

City

Country

Quantity

Phone: Day

E-mail Address

* Add \$32 for each additional shipping address

\section{Payment and Credit Card Details}

Enclosed: Personal Check Credit Card Payment Details

Checks must be paid in U.S. dollars and drawn on a U.S. Bank.

Credit Card: _ VISA _ Am. Exp. _ MasterCard

Card Number

Expiration Date

Signature:

Please send your order form and prepayment made payable to:

\section{Cadmus Reprints}

P.O. Box 751903

Charlotte, NC 28275-1903

Note: Do not send express packages to this location, PO Box. FEIN \#:541274108

Signature

described in this document.

\section{Invoice or Credit Card Information}

Invoice Address Please Print Clearly

Please complete Invoice address as it appears on credit card statement

Name

Institution

Department

Street

City

Country

State

Phone

E-mail Address

Cadmus will process credit cards and Cadmus Journal Services will appear on the credit card statement.

If you don't mail your order form, you may fax it to 410-820-9765 with your credit card information.

Date 


\section{Radiology 2008}

Black and White Reprint Prices

Domestic (USA only)

\begin{tabular}{|c|c|c|c|c|c|c|}
\hline $\begin{array}{c}\text { \# of } \\
\text { Pages }\end{array}$ & $\mathbf{5 0}$ & $\mathbf{1 0 0}$ & $\mathbf{2 0 0}$ & $\mathbf{3 0 0}$ & $\mathbf{4 0 0}$ & $\mathbf{5 0 0}$ \\
\hline $\mathbf{1 - 4}$ & $\$ 221$ & $\$ 233$ & $\$ 268$ & $\$ 285$ & $\$ 303$ & $\$ 323$ \\
\hline $\mathbf{5 - 8}$ & $\$ 355$ & $\$ 382$ & $\$ 432$ & $\$ 466$ & $\$ 510$ & $\$ 544$ \\
\hline $\mathbf{9 - 1 2}$ & $\$ 466$ & $\$ 513$ & $\$ 595$ & $\$ 652$ & $\$ 714$ & $\$ 775$ \\
\hline $\mathbf{1 3 - 1 6}$ & $\$ 576$ & $\$ 640$ & $\$ 749$ & $\$ 830$ & $\$ 912$ & $\$ 995$ \\
\hline $\mathbf{1 7 - 2 0}$ & $\$ 694$ & $\$ 775$ & $\$ 906$ & $\$ 1,017$ & $\$ 1,117$ & $\$ 1,220$ \\
\hline $\mathbf{2 1 - 2 4}$ & $\$ 809$ & $\$ 906$ & $\$ 1,071$ & $\$ 1,200$ & $\$ 1,321$ & $\$ 1,471$ \\
\hline $\mathbf{2 5 - 2 8}$ & $\$ 928$ & $\$ 1,041$ & $\$ 1,242$ & $\$ 1,390$ & $\$ 1,544$ & $\$ 1,688$ \\
\hline $\mathbf{2 9 - 3 2}$ & $\$ 1,042$ & $\$ 1,178$ & $\$ 1,403$ & $\$ 1,568$ & $\$ 1,751$ & $\$ 1,924$ \\
\hline Covers & $\$ 97$ & $\$ 118$ & $\$ 215$ & $\$ 323$ & $\$ 442$ & $\$ 555$ \\
\hline
\end{tabular}

\begin{tabular}{|c|c|c|c|c|c|c|}
\hline \multicolumn{7}{|c|}{ International (includes Canada and Mexico) } \\
\hline $\begin{array}{c}\text { \# of } \\
\text { Pages }\end{array}$ & $\mathbf{5 0}$ & $\mathbf{1 0 0}$ & $\mathbf{2 0 0}$ & $\mathbf{3 0 0}$ & $\mathbf{4 0 0}$ & $\mathbf{5 0 0}$ \\
\hline $\mathbf{1 - 4}$ & $\$ 272$ & $\$ 283$ & $\$ 340$ & $\$ 397$ & $\$ 446$ & $\$ 506$ \\
\hline $\mathbf{5 - 8}$ & $\$ 428$ & $\$ 455$ & $\$ 576$ & $\$ 675$ & $\$ 784$ & $\$ 884$ \\
\hline $\mathbf{9 - 1 2}$ & $\$ 580$ & $\$ 626$ & $\$ 805$ & $\$ 964$ & $\$ 1,115$ & $\$ 1,278$ \\
\hline $\mathbf{1 3 - 1 6}$ & $\$ 724$ & $\$ 786$ & $\$ 1,023$ & $\$ 1,232$ & $\$ 1,445$ & $\$ 1,652$ \\
\hline $\mathbf{1 7}-\mathbf{2 0}$ & $\$ 878$ & $\$ 958$ & $\$ 1,246$ & $\$ 1,520$ & $\$ 1,774$ & $\$ 2,030$ \\
\hline $\mathbf{2 1 - 2 4}$ & $\$ 1,022$ & $\$ 1,119$ & $\$ 1,474$ & $\$ 1,795$ & $\$ 2,108$ & $\$ 2,426$ \\
\hline $\mathbf{2 5 - 2 8}$ & $\$ 1,176$ & $\$ 1,291$ & $\$ 1,700$ & $\$ 2,070$ & $\$ 2,450$ & $\$ 2,813$ \\
\hline $\mathbf{2 9 - 3 2}$ & $\$ 1,316$ & $\$ 1,452$ & $\$ 1,936$ & $\$ 2,355$ & $\$ 2,784$ & $\$ 3,209$ \\
\hline Covers & $\$ 156$ & $\$ 176$ & $\$ 335$ & $\$ 525$ & $\$ 716$ & $\$ 905$ \\
\hline
\end{tabular}

Minimum order is 50 copies. For orders larger than 500 copies, please consult Cadmus Reprints at 800-407-9190.

\section{Reprint Cover}

Cover prices are listed above. The cover will include the publication title, article title, and author name in black.

\section{Shipping}

Shipping costs are included in the reprint prices. Domestic orders are shipped via UPS Ground service. Foreign orders are shipped via a proof of delivery air service.

\section{Multiple Shipments}

Orders can be shipped to more than one location. Please be aware that it will cost $\$ 32$ for each additional location.

\section{Delivery}

Your order will be shipped within 2 weeks of the journal print date. Allow extra time for delivery.

\section{Color Reprint Prices}

\begin{tabular}{|c|c|c|c|c|c|c|}
\hline \multicolumn{7}{|c|}{ Domestic (USA only) } \\
\hline $\begin{array}{c}\text { \# of } \\
\text { Pages }\end{array}$ & $\mathbf{5 0}$ & $\mathbf{1 0 0}$ & $\mathbf{2 0 0}$ & $\mathbf{3 0 0}$ & $\mathbf{4 0 0}$ & $\mathbf{5 0 0}$ \\
\hline $\mathbf{1 - 4}$ & $\$ 223$ & $\$ 239$ & $\$ 352$ & $\$ 473$ & $\$ 597$ & $\$ 719$ \\
\hline $\mathbf{5 - 8}$ & $\$ 349$ & $\$ 401$ & $\$ 601$ & $\$ 849$ & $\$ 1,099$ & $\$ 1,349$ \\
\hline $\mathbf{9 - 1 2}$ & $\$ 486$ & $\$ 517$ & $\$ 852$ & $\$ 1,232$ & $\$ 1,609$ & $\$ 1,992$ \\
\hline $\mathbf{1 3 - 1 6}$ & $\$ 615$ & $\$ 651$ & $\$ 1,105$ & $\$ 1,609$ & $\$ 2,117$ & $\$ 2,624$ \\
\hline $\mathbf{1 7 - 2 0}$ & $\$ 759$ & $\$ 787$ & $\$ 1,357$ & $\$ 1,997$ & $\$ 2,626$ & $\$ 3,260$ \\
\hline $\mathbf{2 1 - 2 4}$ & $\$ 897$ & $\$ 924$ & $\$ 1,611$ & $\$ 2,376$ & $\$ 3,135$ & $\$ 3,905$ \\
\hline $\mathbf{2 5 - 2 8}$ & $\$ 1,033$ & $\$ 1,071$ & $\$ 1,873$ & $\$ 2,757$ & $\$ 3,650$ & $\$ 4,536$ \\
\hline $\mathbf{2 9 - 3 2}$ & $\$ 1,175$ & $\$ 1,208$ & $\$ 2,122$ & $\$ 3,138$ & $\$ 4,162$ & $\$ 5,180$ \\
\hline Covers & $\$ 97$ & $\$ 118$ & $\$ 215$ & $\$ 323$ & $\$ 442$ & $\$ 555$ \\
\hline
\end{tabular}

\begin{tabular}{|c|c|c|c|c|c|c|}
\hline \multicolumn{7}{|c|}{ International (includes Canada and Mexico)) } \\
\hline $\begin{array}{c}\text { \# of } \\
\text { Pages }\end{array}$ & $\mathbf{5 0}$ & $\mathbf{1 0 0}$ & $\mathbf{2 0 0}$ & $\mathbf{3 0 0}$ & $\mathbf{4 0 0}$ & $\mathbf{5 0 0}$ \\
\hline $\mathbf{1 - 4}$ & $\$ 278$ & $\$ 290$ & $\$ 424$ & $\$ 586$ & $\$ 741$ & $\$ 904$ \\
\hline $\mathbf{5 - 8}$ & $\$ 429$ & $\$ 472$ & $\$ 746$ & $\$ 1,058$ & $\$ 1,374$ & $\$ 1,690$ \\
\hline $\mathbf{9 - 1 2}$ & $\$ 604$ & $\$ 629$ & $\$ 1,061$ & $\$ 1,545$ & $\$ 2,011$ & $\$ 2,494$ \\
\hline $\mathbf{1 3 - 1 6}$ & $\$ 766$ & $\$ 797$ & $\$ 1,378$ & $\$ 2,013$ & $\$ 2,647$ & $\$ 3,280$ \\
\hline $\mathbf{1 7 - 2 0}$ & $\$ 945$ & $\$ 972$ & $\$ 1,698$ & $\$ 2,499$ & $\$ 3,282$ & $\$ 4,069$ \\
\hline $\mathbf{2 1 - 2 4}$ & $\$ 1,110$ & $\$ 1,139$ & $\$ 2,015$ & $\$ 2,970$ & $\$ 3,921$ & $\$ 4,873$ \\
\hline $\mathbf{2 5 - 2 8}$ & $\$ 1,290$ & $\$ 1,321$ & $\$ 2,333$ & $\$ 3,437$ & $\$ 4,556$ & $\$ 5,661$ \\
\hline $\mathbf{2 9 - 3 2}$ & $\$ 1,455$ & $\$ 1,482$ & $\$ 2,652$ & $\$ 3,924$ & $\$ 5,193$ & $\$ 6,462$ \\
\hline Covers & $\$ 156$ & $\$ 176$ & $\$ 335$ & $\$ 525$ & $\$ 716$ & $\$ 905$ \\
\hline
\end{tabular}

\section{Tax Due}

Residents of Virginia, Maryland, Pennsylvania, and the District of Columbia are required to add the appropriate sales tax to each reprint order. For orders shipped to Canada, please add 7\% Canadian GST unless exemption is claimed.

\section{Ordering}

Reprint order forms and purchase order or prepayment is required to process your order. Please reference journal name and reprint number or manuscript number on any correspondence. You may use the reverse side of this form as a proforma invoice. Please return your order form and prepayment to:

\section{Cadmus Reprints \\ P.O. Box 751903 \\ Charlotte, NC 28275-1903}

Note: Do not send express packages to this location, PO Box. FEIN \#:541274108

Please direct all inquiries to:

Rose A. Baynard

800-407-9190 (toll free number) 410-819-3966 (direct number) 410-820-9765 (FAX number) baynardr@cadmus.com (e-mail)
Reprint Order Forms and purchase order or prepayments must be received 72 hours after receipt of form. 